\title{
Eficiencia de la biodegradación de hidrocarburos de petróleo por hongos filamentosos aislados de suelo contaminado
}

\section{Efficiency of biodegradation of petroleum hydrocarbons by filamentous fungi isolated from contaminated soil}

\author{
*Hans Contreras ${ }^{1}$, Carmen Carreño ${ }^{2}$
}

\begin{abstract}
RESUMEN
La biodegradación disminuye el impacto ambiental negativo de los derrames de hidrocarburos de petróleo. El objetivo de esta investigación fue determinar la eficiencia de biodegradación de hidrocarburos de petróleo de diez cultivos de hongos filamentosos hidrocarbonoclásticos seleccionados. Las muestras de suelo contaminado con hidrocarburos de petróleo presentaron concentración de hidrocarburos totales (HTP) (25 $\left.987 \mathrm{mg} \mathrm{kg}^{-1}\right)$, microorganismos totales $\left(>1,1 \times 10^{7} \mathrm{NMP} \mathrm{g}^{-1}\right)$ y microorganismos hidrocarbonoclásticos $\left(1,1 \times 10^{6} \mathrm{NMP} \mathrm{g}^{-1}\right)$ y un nivel de toxicidad severo en el índice de germinación de Raphanus sativus L. "rabanito". Los hongos filamentosos se aislaron en agar Bushnell Haas-petróleo 1\%, obteniéndose 221 aislados, identificándose 14 géneros: Cunninghamella, Penicillium, Paecilomyces, Rhizopus, Syncephalastrum, Periconia, Fusarium, Bipolaris, Monilia, Cladosporium, Gliocladium, Aspergillus, Memnoniella y Helminthosporium. Todos los géneros a excepción de Helminthosporium demostraron ser hidrocarbonoclásticos y se seleccionaron aquellos que alcanzaron los mayores valores de biomasa (1,00 a 1,95 g). En el suelo tratado con Aspergillus sp. HP-031 se alcanzó el mayor índice de germinación desde los 60 días, la disminución de la toxicidad a un nivel bajo a los 90 días y la mayor eficiencia (73\%) en la degradación del HTP, demostrándose el potencial de este hongo para la biorremediación.
\end{abstract}

Palabras clave: Biodegradación, hongos filamentosos, hidrocarbonoclásticos, petróleo.

\begin{abstract}
Biodegradation reduces the negative environmental impact of oil spills. The objective of this research was to determine the efficiency of the biodegradation of petroleum hydrocarbons by ten cultures of hydrocarbonoclastic filamentous fungi selected. Samples of soil contaminated with petroleum hydrocarbons had a content of HTP $(25$ $\left.987 \mathrm{mg} \mathrm{kg}^{-1}\right)$, total microorganisms $\left(>1,1 \times 10^{7} \mathrm{NMP} \mathrm{g}^{-1}\right)$ and hydrocarbonoclastic microorganisms $\left(1,1 \times 10^{6} \mathrm{NMP}\right.$ $\left.\mathrm{g}^{-1}\right)$ and a level of severe toxicity in the germination index of Raphanus sativus L. "rabanito". Filamentous fungi were isolated on agar Bushnell Haas-oil 1\%, obteined 221 isolates, identified 14 genera: Cunninghamella, Penicillium y Paecilomyces, Rhizopus, Syncephalastrum, Periconia, Fusarium, Bipolaris, Monilia, Cladosporium, Gliocladium, Memnoniella y Helminthosporium. All genera with the exception of Helminthosporium were shown to be hydrocarbonoclastic and and those that reached the highest values of biomass (1.00 to $1.95 \mathrm{~g}$ ) were selected. In soil treated with Aspergillus sp. HP-031 achieved the highest germination index from 60 days, decreased toxicity at a low level in the 90 days and the highest efficiency $(73 \%)$ in the degradation of HTP, demonstrating the potential of this fungus for the Bioremediation.
\end{abstract}

Keywords: Biodegradation, filamentous fungi, hydrocarbonoclastic, petroleum.

\footnotetext{
${ }^{1}$ * Biólogo. Universidad Nacional Pedro Ruiz Gallo. Correo electrónico: hans94cm@gmail.com

${ }^{2}$ Bióloga. Universidad Nacional Pedro Ruiz Gallo. Correo electrónico: c.carrenof1@hotmail.com
} 


\section{INTRODUCCIÓN}

La explotación, producción, refinación y transporte de petróleo y sus derivados conlleva ocasionalmente accidentes técnicos y operacionales que causan severo deterioro al ambiente y daños irreversibles a los ecosistemas, afectando la flora, fauna y la composición de las comunidades microbianas autóctonas (Pernía et al., 2012).

Debido al impacto ambiental negativo de los hidrocarburos de petróleo, se han desarrollado técnicas para su eliminación, entre los que se consideran la extracción de hidrocarburos por vacío, lavado del suelo contaminado con agua, incineración, recuperación electrocinética, entre otras. Con estas técnicas se consiguen resultados positivos; pero su elevado costo económico, es un obstáculo para su empleo. En este contexto, la biorremediación, es una alternativa viable para disminuir el deterioro de la calidad del suelo por el derrame de petróleo (Cabanillas y Pissani, 2015).

La biorremediación consiste en el uso de plantas y microorganismos naturales (Galindo \& Llontop, 2015) o modificados genéticamente para neutralizar los contaminantes, disminuyendo o eliminando su toxicidad para los seres vivos. Entre los microorganismos, los hongos filamentosos son capaces de mineralizar los hidrocarburos de petróleo hasta dióxido de carbono y agua (Tapia, 2002); sin embargo, su proliferación y actividad puede ser afectada por la población muy baja. Por esta razón, se requiere aislar hongos filamentosos en zonas impactadas, para caracterizarlos, seleccionarlos, propagarlos y posteriormente reintroducirlos, asegurando al máximo su eficiencia en la biodegradación de los hidrocarburos de petróleo y biorremediación de los suelos contaminados.

Los lugares contaminados con petróleo, en el distrito de Puerto Eten, región Lambayeque, tienen una diversidad biológica que no ha sido investigada (Llanos, 2012), para la búsqueda de hongos filamentosos degradadores de hidrocarburos de petróleo. Por lo que se planteó como objetivo determinar la eficiencia de biodegradación de hidrocarburos de petróleo por diez cultivos seleccionados de hongos filamentosos hidrocarbonoclásticos.

\section{II.MATERIAL Y MÉTODOS}

La investigación se realizó en dos fases. En la primera fase (descriptiva) se determinaron las características químicas, microbiológicas y toxicidad del suelo experimental contaminado con hidrocarburos de petróleo, se aislaron e identificaron hongos filamentosos y se seleccionaron los hongos hidrocarbonoclásticos. En la segunda fase (explicativa) se determinó la eficiencia de la biodegradación de hidrocarburos de petróleo por diez cultivos de hongos filamentosos hidrocarbonoclásticos seleccionados. La hipótesis en la primera fase se contrastó con el diseño no experimental "Sólo Después" y en la segunda fase con el diseño experimental completamente aleatorio, DCA (Hernández et al., 2014). Los tratamientos fueron 12, correspondientes a T1: Testigo absoluto (agua destilada), T2: Testigo abiótico (formaldehido), T3 a T12: cultivos de hongos filamentosos hidrocarbonoclásticos.

En la fase descriptiva, la población estuvo constituida por el suelo contaminado con hidrocarburos de petróleo en el distrito de Puerto Eten, región Lambayeque y se investigaron 54 muestras probabilísticas de suelo colectadas durante noviembre a diciembre de 2016. El número de muestras fue calculado (Hernández et al., 2014) tomando en cuenta una prevalencia de $90 \%$, determinada mediante un muestreo piloto. En la fase explicativa, la población fueron los hongos hidrocarbonoclásticos aislados del suelo contaminado durante noviembre a diciembre de 2016 y la muestra no probabilística y por conveniencia estuvo constituida por diez cultivos de hongos.

Las muestras de suelo contaminado, de aproximadamente $1 \mathrm{~kg}$, se obtuvieron con una palana, eliminando los $2 \mathrm{~cm}$ superficiales y colectando el suelo a una profundidad de $10 \mathrm{~cm}$ (Llanos, 2012) en 54 puntos de muestreo ubicados aleatoriamente. El análisis químico, microbiológico y toxicidad del suelo contaminado se realizó en submuestras de $1 \mathrm{~kg}$.

La concentración de hidrocarburos totales (HTP), se determinó mediante cromatografía de gases acoplada a espectrometría de masas (GC-MS), utilizando un cromatógrafo de gases modelo $6890 \mathrm{~N}$ (Net Work GC system) y un espectrómetro de masas modelo 5975 inert XL(Agilent Tecnologies).

En el análisis microbiológico de tres submuestras de suelo de 10 g cada una, se determinó el número de microorganismos totales

microorganismos hidrocarbonoclásticos, mediante la técnica del número más probable (Llanos, 2012). Los tubos se incubaron a $30^{\circ} \mathrm{C}$ por 10 días y la turbidez del medio de cultivo se consideró positivo a 1 a presencia de microorganismos totales e hidrocarbonoclásticos, realizándose el cálculo 
correspondiente según el método estándar.

La toxicidad de los contaminantes del suelo experimental se investigó por triplicado en submuestras de $10 \mathrm{~g}$, utilizando semillas de Raphanus sativus L. "rabanito" a las que previamente se les determinó el porcentaje de germinación (Flores \& Benites, 2015). En placas de Petri, se depositaron las submuestras de suelo, se humedecieron con $12 \mathrm{~mL}$ de agua destilada y en cada placa, con una pinza se depositaron 25 semillas de rabanito. Todas las placas se cubrieron con papel metálico durante $\quad 120 \mathrm{~h}, \mathrm{a} 30^{\circ} \mathrm{C}$ y a las 120 $\mathrm{h}$ se contaron las semillas germinadas y se midió la longitud de las radículas emergidas, calculando (Llanos, 2012) el porcentaje relativo de germinación (PGR), crecimiento relativo de la radícula (CRR) e índice de germinación (IG):

$\mathrm{PGR}=\frac{\mathrm{N}^{\circ} \text { de semillas germinadas en el suelo contaminado }}{\mathrm{N}^{\circ} \text { de semillas germinadas en el testigo }} \times 100$

$\mathrm{CRR}=\frac{\text { Long. } \text { promedio de radículas en el suelo contaminado }}{\text { Long. promedio de radículas en el testigo }} \times 100$

$\mathrm{IG}=\frac{\text { PGR } \times \text { CRR }}{100}$

La fitotoxicidad se determinó con el criterio de interpretación: $\mathrm{IG} \geq 80 \%$ indica que no hay sustancias fitotóxicas o están en muy baja concentración; $80 \%>$ IG $>50 \%$ se interpreta como presencia moderada de estas sustancias y un $\mathrm{IG} \leq$ $50 \%$ indica fuerte presencia de sustancias fitotóxicas, criterios correspondientes a niveles de fitotoxicidad bajo, moderado y severo (Purisaca \& Quevedo, 2015).

\section{Aislamiento, identificación y selección de hongos hidrocarbonoclásticos}

Los hongos filamentosos cultivables de las muestras de suelo contaminado, se enriquecieron, depositando $10 \mathrm{~g}$ de cada muestra en $90 \mathrm{~mL}$ de caldo Bushnell Haas e incubando a $30^{\circ} \mathrm{C}$, por 72 horas, con agitación manual diaria durante 10 minutos (Galindo \& Llontop, 2015). Después de la incubación, se tomaron alícuotas de $0,1 \mathrm{~mL}$ y se sembraron mediante la técnica de agotamiento y estría en agar Bushnell Haas con $1 \mathrm{~mL}(1 \%)$ de petróleo crudo, incubándose a $30^{\circ} \mathrm{C}$ hasta por 10 días. Las colonias de hongos desarrollados se agruparon según sus características macroscópicas y la identificación se realizó en cultivos monospóricos (Arias \& Piñeros, 2008), con ayuda de las claves taxonómicas de Seifert (1996). Los hongos filamentosos se consideraron hidrocarbonoclásticos cuando utilizaron el contaminante como fuente de carbono y energía (Llanos, 2012), desarrollando micelio observable, con y sin viraje del indicador. El inóculo de los hongos se obtuvo con cultivos de 10 días de crecimiento en PDA con $1 \%$ de petróleo a los que se les agregaron $10 \mathrm{~mL}$ de solución salina esterilizada $(\mathrm{NaCl} 0,85 \%$ p/v) para obtener una suspensión de conidios, que se filtró en gasa esterilizada y su concentración se estandarizó a $10^{4}$ conidios $\mathrm{mL}^{-1}$ mediante recuento de células en la cámara de Neubauer (Corrales et al., 2012).

El inóculo fue cultivado por triplicado en $5 \mathrm{~mL}$ de caldo Bushnell Haas con $1 \%$ de petróleo a $30^{\circ} \mathrm{C}$, con agitación manual diaria por $10 \mathrm{~min}$, registrándose los días requeridos para la observación del micelio y viraje del indicador. Transcurridos 10 días el medio de cultivo, se llevó al autoclave $\left(121^{\circ} \mathrm{C}, 15 \mathrm{lbs}, 20^{\prime}\right)$, se filtró y la biomasa obtenida se deshidrató en estufa a $70^{\circ} \mathrm{C}$, hasta alcanzar peso constante (Tapia, 2002), que fue determinado en una balanza de precisión (EXCELL, 0,01-150 g), seleccionándose los diez cultivos de hongos con los mayores valores.

\section{Biodegradación de hidrocarburos de petróleo}

El suelo experimental contaminado con petróleo se distribuyó en 36 bandejas de polipropileno a razón de $1,20 \mathrm{~kg}$ por cada una de ellas. A continuación, siguiendo el procedimiento descrito por Corrales et al. (2012), se depositaron $120 \mathrm{~mL}$ del inóculo del hongo correspondiente por bandeja, al testigo absoluto se agregó agua destilada y al testigo abiótico formaldehido al 5\%. Inmediatamente después de la inoculación se tomaron submuestras de $10 \mathrm{~g}$ para el análisis microbiológico (Llanos, 2012) y el ensayo de toxicidad en rabanito (Purisaca \& Quevedo, 2015). Las bandejas se mantuvieron en el invernadero a temperatura ambiental, removiéndolas con una palana de mano durante 5 min y regándolas con agua declorada según el requerimiento. Cada 30 días, hasta por 90 días, se tomaron submuestras para el análisis microbiológico y ensayo de toxicidad respectivo. A los 90 días, se seleccionó el suelo del tratamiento con el que se alcanzó el mayor índice de germinación de rabanito, correspondiente a la menor toxicidad, se determinó la concentración de HTP y se calculó la eficiencia de la biodegradación (Llanos, 2012) de petróleo mediante la siguiente fórmula:

$$
\mathrm{E}=\frac{\mathrm{Si}-\mathrm{Sf}}{\mathrm{Si}} \times 100
$$

Donde:

$\mathrm{E}=$ Eficiencia de biodegradación de petróleo (\%)

$\mathrm{Si}=$ Concentración inicial de TPH

$\mathrm{Sf}=$ Concentración final de $\mathrm{TPH}$ 


\section{Análisis estadístico de los datos}

Se realizó el análisis de varianza de los valores del índice de germinación y la superioridad entre los tratamientos se determinó mediante la prueba múltiple de Tukey con un nivel de significancia de 0,05 (Hernández et al., 2014), utilizando los programas Microsoft Office Word y Excel versión 2013.

\section{RESULTADOS}

El suelo contaminado presentó HTP de $25987 \mathrm{mg}$ $\mathrm{kg}^{-1}$. La carga microbiana fue $>1,1 \times 10^{7} \mathrm{NMP} \mathrm{g}^{-1}$, población que superó a la hidrocarbonoclástica de $1,1 \times 10^{6} \mathrm{NMP} \mathrm{g}^{-1}$. En cuanto al nivel de toxicidad fue severo en el índice de germinación de rabanito.

En todas las muestras de suelo contaminado con hidrocarburos de petróleo se aislaron hongos filamentosos, obteniéndose 221 aislados. Se identificaron 14 géneros, siendo los más frecuentes, Aspergillus (20,8\%), Cunninghamella (12,6\%) Penicillium y Paecilomyces (10,8\%), seguidos de Rhizopus, Syncephalastrum, Periconia, Fusarium, Bipolaris, Monilia y Cladosporium (8,5 a $2,7 \%)$. Los géneros menos frecuentes fueron Gliocladium, Memnoniella y Helminthosporium (2,2\%).

El 98\% de los hongos filamentosos aislados e identificados se consideraron hidrocarbonoclásticos porque utilizaron el petróleo como fuente de carbono y energía observándose micelio con o sin viraje del indicador al amarillo. El 2\% de los hongos filamentosos del género Helminthosporium no demostraron ser hidrocarbonoclásticos.

Los hidrocarbonoclásticos desarrollaron micelio observable en el caldo Bushnell Haas con petróleo como fuente de carbono y energía durante 48 a 168 h, correspondiendo el mayor porcentaje (35\%) a $96 \mathrm{~h} \mathrm{y}$ el menor porcentaje (7\%) a $144 \mathrm{~h}$. En el 58\% del caldo Bushnell Haas con petróleo cultivado se observó viraje del indicador púrpura de bromocresol al amarillo en un tiempo que osciló entre 24 a 96 h y

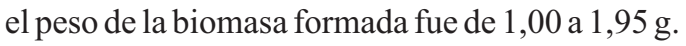

Los hongos filamentosos hidrocarbonoclásticos seleccionados fueron los que alcanzaron la mayor biomasa después de 10 días de incubación, correspondiendo a Aspergillus sp. 024 (1,95 g), Penicillium sp. 029 (1,91 g), Bipolaris sp. 016 (1,84 g), Aspergillus sp. 031 (1,83 g), Aspergillus sp. 015 (1,82 g), Cunninghamella sp. 018 (1,81 g), Cunninghamella sp. 025 (1,80 g), Periconia sp. 030 $(1,79 \mathrm{~g})$, Aspergillus sp. $017(1,78 \mathrm{~g})$ y Paecilomyces sp. $023(1,78 \mathrm{~g})$.
En el proceso de biodegradación los microorganismos se incrementaron, alcanzando la máxima población de heterótrofos totales a los 30 días (Tabla 1) y de hidrocarbonoclásticos a los 60 días (Tabla 2). En los testigos abiótico y absoluto, las máximas poblaciones se alcanzaron a los 60 días. En todos los tratamientos los microorganismos disminuyeron a los 90 días.

Tabla 1. Población de microorganismos totales (NMP $\mathrm{g}^{-1}$ ) durante la biodegradación de hidrocarburos de petróleo en suelo contaminado

\begin{tabular}{|c|c|c|c|c|c|}
\hline & \multirow{2}{*}{ Tratamientos } & \multicolumn{4}{|c|}{$\mathrm{NMP} \mathrm{g}^{-1} /$ días } \\
\hline & & 0 & 30 & 60 & 90 \\
\hline T1 & $\begin{array}{l}\text { Aspergillus sp. } \\
\text { HP-015 }\end{array}$ & $1,1 \times 10^{7}$ & $>_{1,1 \times 10^{7}}$ & $1,1 \times 10^{7}$ & $1,1 \times 10^{7}$ \\
\hline T2 & $\begin{array}{l}\text { Bipolaris sp. } \\
\text { HP-016 }\end{array}$ & $2,1 \times 10^{5}$ & $1,1 \times 10^{7}$ & $2,4 \times 10^{7}$ & $2,4 \times 10^{6}$ \\
\hline T3 & $\begin{array}{l}\text { Aspergillus sp. } \\
\text { HP-017 }\end{array}$ & $2,1 \times 10^{6}$ & $>1,1 \times 10^{7}$ & $1,1 \times 10^{7}$ & $2,4 \times 10^{6}$ \\
\hline T4 & $\begin{array}{l}\text { Cunninghamella sp. } \\
\text { HP-018 }\end{array}$ & $1,1 \times 10^{7}$ & $>1,1 \times 10^{7}$ & $4,6 \times 10^{6}$ & $2,1 \times 10^{6}$ \\
\hline T5 & $\begin{array}{l}\text { Paecilomyces sp. } \\
\text { HP-023 }\end{array}$ & $1,5 \times 10^{6}$ & $>1,1 \times 10^{7}$ & $1,1 \times 10^{7}$ & $4,6 \times 10^{6}$ \\
\hline T6 & $\begin{array}{l}\text { Aspergillus sp. } \\
\text { HP-024 }\end{array}$ & $1,1 \times 10^{7}$ & $>1,1 \times 10^{7}$ & $4,6 \times 10^{6}$ & $2,1 \times 10^{6}$ \\
\hline $\mathrm{T7}$ & $\begin{array}{l}\text { Cunninghamella sp. } \\
\text { HP-0125 }\end{array}$ & $1,1 \times 10^{7}$ & $>1,1 \times 10^{7}$ & $2,1 \times 10^{6}$ & $2,4 \times 10^{6}$ \\
\hline T8 & $\begin{array}{l}\text { penicillium sp. } \\
\text { HP-029 }\end{array}$ & $1,1 \times 10^{7}$ & $>1,1 \times 10^{7}$ & $4,6 \times 10^{6}$ & $2,1 \times 10^{6}$ \\
\hline т9 & $\begin{array}{l}\text { Periconia sp. } \\
\text { HP-030 }\end{array}$ & $2,9 \times 10^{6}$ & $1,1 \times 10^{7}$ & $1,5 \times 10^{6}$ & $4,3 \times 10^{5}$ \\
\hline $\mathrm{T} 10$ & $\begin{array}{l}\text { Aspergillus sp. } \\
\text { HP-031 }\end{array}$ & $1,1 \times 10^{7}$ & $>1,1 \times 10^{7}$ & $1,1 \times 10^{7}$ & $4,6 \times 10^{6}$ \\
\hline T11 & Testigo Absoluto & $3,5 \times 10^{5}$ & $2,3 \times 10^{5}$ & $9,3 \times 10^{5}$ & $2,3 \times 10^{5}$ \\
\hline T12 & Testigo Abiótico & $7,3 \times 10^{4}$ & $1,5 \times 10^{5}$ & $3,9 \times 10^{5}$ & $2,8 \times 10^{5}$ \\
\hline
\end{tabular}

Los índices de germinación de rabanito en el suelo biodegradado y con los testigos se incrementaron conforme transcurrió el tiempo y por el contrario, los niveles de toxicidad disminuyeron. A los 90 días, los mayores valores en el índice de germinación significativamente iguales correspondieron a Aspergillus spp. HP-031 y 017 (Tabla 3).

HP-031 se alcanzó el mayor índice de germinación desde los 30 días, la disminución de la toxicidad a un nivel bajo a los 90 días y una eficiencia de $73 \%$ en la degradación del HTP(Figura 1, Tabla 4). 
Tabla 2. Población de microorganismos hidrocarbonoclásticos (NMP $\mathrm{g}^{-1}$ ) durante la biodegradación de hidrocarburos de petróleo en suelo contaminado

\begin{tabular}{|c|c|c|c|c|c|}
\hline & \multirow{2}{*}{ Tratamientos } & \multicolumn{4}{|c|}{$\mathrm{NMP} \mathrm{g}^{-1} /$ días } \\
\hline & & 0 & 30 & 60 & 90 \\
\hline T1 & $\begin{array}{l}\text { Aspergillus sp. } \\
\text { HP-015 }\end{array}$ & $2,4 \times 10^{5}$ & $4,6 \times 10^{5}$ & $>1,1 \times 10^{6}$ & $4,6 \times 10^{5}$ \\
\hline T2 & $\begin{array}{l}\text { Bipolaris sp. } \\
\text { HP-016 }\end{array}$ & $5,3 \times 10^{4}$ & $6,4 \times 10^{4}$ & $>1,1 \times 10^{6}$ & $2,4 \times 10^{5}$ \\
\hline T3 & $\begin{array}{l}\text { Aspergillus sp. } \\
\text { HP-017 }\end{array}$ & $2,9 \times 10^{5}$ & $4,6 \times 10^{5}$ & $>1,1 \times 10^{6}$ & $2,9 \times 10^{5}$ \\
\hline T4 & $\begin{array}{l}\text { Cunninghamella sp. } \\
\text { HP-018 }\end{array}$ & $2,4 \times 10^{5}$ & $1,1 \times 10^{6}$ & $>1,1 \times 10^{6}$ & $2,1 \times 10^{5}$ \\
\hline T5 & $\begin{array}{l}\text { Paecilomyces sp. } \\
\text { HP-023 }\end{array}$ & $3,5 \times 10^{4}$ & $2,9 \times 10^{5}$ & $>1,1 \times 10^{6}$ & $2,1 \times 10^{5}$ \\
\hline T6 & $\begin{array}{l}\text { Aspergillus sp. } \\
\text { HP-024 }\end{array}$ & $2,4 \times 10^{5}$ & $1,1 \times 10^{6}$ & $>1,1 \times 10^{6}$ & $4,3 \times 10^{4}$ \\
\hline T7 & $\begin{array}{l}\text { Cunninghamella sp. } \\
\text { HP-0125 }\end{array}$ & $2,4 \times 10^{5}$ & $4,6 \times 10^{5}$ & $>1,1 \times 10^{6}$ & $3,5 \times 10^{4}$ \\
\hline T8 & $\begin{array}{l}\text { penicillium sp. } \\
\text { HP-029 }\end{array}$ & $2,9 \times 10^{5}$ & $4,6 \times 10^{5}$ & $>1,1 \times 10^{6}$ & $4,3 \times 10^{4}$ \\
\hline T9 & $\begin{array}{l}\text { Periconia sp. } \\
\text { HP-030 }\end{array}$ & $2,4 \times 10^{5}$ & $1,1 \times 10^{6}$ & $>1,1 \times 10^{6}$ & $4,3 \times 10^{5}$ \\
\hline T10 & $\begin{array}{l}\text { Aspergillus sp. } \\
\text { HP-031 }\end{array}$ & $2,4 \times 10^{5}$ & $4,6 \times 10^{5}$ & $>1,1 \times 10^{6}$ & $2,9 \times 10^{5}$ \\
\hline T11 & Testigo Absoluto & $3,5 \times 10^{4}$ & $5,3 \times 10^{4}$ & $2,1 \times 10^{5}$ & $2,3 \times 10^{4}$ \\
\hline T12 & Testigo Abiótico & $2,0 \times 10^{4}$ & $2,7 \times 10^{4}$ & $1,2 \times 10^{5}$ & $2,1 \times 10^{4}$ \\
\hline
\end{tabular}

Tabla 3: Índice de germinación de Raphanus sativus L. durante la biodegradación en el suelo biodegradado con Aspergillus sp.

\begin{tabular}{|c|c|c|c|c|c|c|}
\hline & \multirow{2}{*}{ Tratamientos } & \multicolumn{4}{|c|}{$\mathrm{NMP} \mathrm{g}^{-1} /$ días } & \multirow{2}{*}{$\begin{array}{c}\text { Sig. } \\
(\alpha=0,05)\end{array}$} \\
\hline & & 0 & 30 & 60 & 90 & \\
\hline T1 & $\begin{array}{l}\text { Aspergillus sp. } \\
\text { HP-015 }\end{array}$ & $2-S$ & $12-\mathrm{s}$ & $64-\mathrm{M}$ & 73-M & b \\
\hline T2 & $\begin{array}{l}\text { Bipolaris sp. } \\
\text { HP-016 }\end{array}$ & $3-5$ & $10-5$ & $42-5$ & $66-M$ & c \\
\hline T3 & $\begin{array}{l}\text { Aspergillus sp. } \\
\text { HP-017 }\end{array}$ & $2-S$ & $11-5$ & $60-M$ & 79-M & a \\
\hline T4 & $\begin{array}{l}\text { Cunninghamella sp. } \\
\text { HP-018 }\end{array}$ & $4-S$ & 8-S & $48-S$ & $72-M$ & $b$ \\
\hline T5 & $\begin{array}{l}\text { Paecilomyces sp. } \\
\text { HP-023 }\end{array}$ & $3-5$ & $8-S$ & $40-5$ & $55-\mathrm{M}$ & $\mathrm{cd}$ \\
\hline T6 & $\begin{array}{l}\text { Aspergillus sp. } \\
\text { HP-024 }\end{array}$ & $3-S$ & 9-S & $50-5$ & $68-M$ & bc \\
\hline $\mathrm{T7}$ & $\begin{array}{l}\text { Cunninghamella sp. } \\
\text { HP-0125 }\end{array}$ & $3-5$ & $7-5$ & $48-5$ & $70-M$ & bc \\
\hline T8 & $\begin{array}{l}\text { penicillium sp. } \\
\text { HP-029 }\end{array}$ & $2-S$ & $11-S$ & $49-5$ & $72-M$ & $b$ \\
\hline T9 & $\begin{array}{l}\text { Periconia sp. } \\
\text { HP-030 }\end{array}$ & $2-5$ & $9-5$ & $500-5$ & 73-M & b \\
\hline T10 & $\begin{array}{l}\text { Aspergillus sp. } \\
\text { HP-031 }\end{array}$ & $3-5$ & $15-\mathrm{S}$ & $75-M$ & $82-B$ & a \\
\hline T11 & Testigo Absoluto & $3-5$ & $5-S$ & $32-5$ & $45-S$ & e \\
\hline T12 & Testigo Abiótico & $0-S$ & $2-S$ & $20-5$ & $31-5$ & $f$ \\
\hline
\end{tabular}

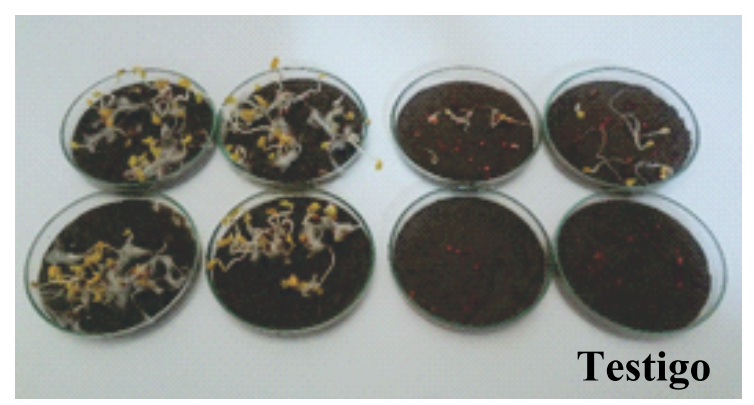

Figura 1. Ensayo de toxicidad en Raphanus sativus L., de suelo biodegradado con Aspergillus sp. HP-031

Tabla 4. Características del suelo biodegradado por 90 días con Aspergillus sp. HP-031

\begin{tabular}{|c|c|c|c|c|}
\hline \multirow{2}{*}{ Características } & \multicolumn{4}{|c|}{ Días } \\
\hline & 0 & 30 & 60 & 90 \\
\hline $\begin{array}{l}\text { Microorganismos } \\
\text { totales (NMP g- }{ }^{1} \text { ) }\end{array}$ & $1,1 \times 10^{7}$ & $>1,1 \times 10^{7}$ & $1,1 \times 10^{7}$ & $4,6 \times 10^{6}$ \\
\hline $\begin{array}{l}\text { Microorganismos } \\
\text { hidrocarbonoclástic } \\
\left.\text { os (NMP g- }{ }^{1}\right)\end{array}$ & $2,4 \times 10^{5}$ & $4,6 \times 10^{5}$ & $>1,1 \times 10^{6}$ & $2,9 \times 10^{5}$ \\
\hline $\begin{array}{l}\text { Indice de } \\
\text { germinación (\%) }\end{array}$ & 3 & 15 & 75 & 82 \\
\hline Nivel de toxicidad & Severo & Severo & Moderado & Bajo \\
\hline HTP (mg gk- $\left.{ }^{1}\right)$ & 25987 & & & 7016 \\
\hline $\begin{array}{l}\text { Eficiencia de } \\
\text { biorremediación } \\
(\%) \\
\end{array}$ & & & & 73 \\
\hline
\end{tabular}

\section{DISCUSIÓN}

La concentración de TPH del suelo investigado fue superior al rango de $19000-25000 \mathrm{mg} \mathrm{kg}^{-1}$ registrado por Hernández et al. (2003) y Purisaca \& Quevedo (2015). La fracción TPH se define como aquellos hidrocarburos del extracto orgánico total pertenecientes a las fracciones saturada y aromática (Llanos, 2012), cuya concentración sirve como indicador del tipo de contaminación. Al respecto, Buendía (2012) mencionó que para biorremediar un suelo, el TPH debe ser menor de $50000 \mathrm{mg} \mathrm{kg}^{-1}$.

Los hongos aislados en el suelo contaminado son considerados heterótrofos tolerantes a los hidrocarburos de petróleo (Rivera et al., 2002), entre los que se encuentran los degradadores o hidrocarbonoclásticos.

Los hongos aislados se identificaron fenotípicamente tomando en cuenta la morfología de la colonia, micelio y estructuras reproductivas, coincidiendo con Vanishree et al. (2014), Chaudhry et al. (2012) y Hasan et al. (2016). En los géneros de hongos aislados del suelo contaminado predominaron Aspergillus, Penicillium, Paecilomyces y 
Cunninghamella, considerados por Pernía et al. (2012) como representativos en sustratos contaminados con crudo y derivados.

La capacidad para formar micelio observable en el caldo Bushnell Haas con petróleo como única fuente de carbono y energía fue el criterio determinante para considerar a un hongo hidrocarbonoclástico, coincidiendo con Rivera et al. (2002). El viraje del indicador al amarillo por la acidez generada también se utilizó como criterio para calificar a los hongos hidrocarbonoclásticos. El indicador es reducido por los productos resultantes de la oxidación de los hidrocarburos de petróleo (Chaudhry et al., 2012).

Los géneros de hongos caracterizados como hidrocarbonoclásticos fueron reconocidos previamente por Arias \& Piñeros (2008) (Paecilomyces, Rhizopus, Penicillium); Chaudhry et al. (2012) (Fusarium, Cladosporium), Pernía et al. (2012) (Cunninghamella, Gliocladium, Monilia Syncephalastrum), Sandhu et al., (2016) (Periconia); Tapia (2002) (Memnoniella) y Deli (2014) (Bipolaris).

Los hongos de los géneros Aspergillus, Cunninghamella, Bipolaris, Paecilomyces y Penicillium fueron seleccionados por la mayor biomasa. La capacidad de estos géneros para la degradación eficiente de hidrocarburos de petróleo y derivados también fue reportada por Chaudhry et al. (2012) (Aspergillus, Penicillium) y Rivera et al. (2002) (Paecilomyces). Por su parte, Pernía et al. (2012) consideran entre otros a Aspergillus en el grupo de hongos con mayor capacidad (52\%) para reducir los niveles de TPH, Penicillium y Paecilomyces en el grupo de capacidad intermedia (27\%) y Cunninghamella en el grupo con menor capacidad degradativa (13\%).

El incremento de los microorganismos en el proceso de biodegradación, se atribuye a que el hidrocarburo como fuente de carbono puede sostener la población microbiana (Hernández et al., 2003). El mayor índice de germinación y menor toxicidad alcanzados con Aspergillus sp. HP-031 se relacionaron con el 73\% de degradación en el HTP. Este género es considerado en el grupo de los hongos con mayor capacidad para reducir los niveles de TPH (Pernía et al., 2012) y su eficiencia fue demostrada previamente por Lotfinasabasl et al. (2012), Hasan (2014) y Hasan et al. (2016).

\section{CONCLUSIONES}

En un suelo contaminado con hidrocarburos de petróleo $\left(\mathrm{TPH}=25987 \mathrm{mg} \mathrm{kg}^{-1}\right)$ se aislaron hongos hidrocarbonoclásticos, destacando Aspergillus sp. HP-031 por la mayor eficiencia en la biodegradación, demostrada por la menor toxicidad del suelo tratado y la disminución (73\%) de la concentración de HTP.

\section{REFERENCIAS BIBLIOGRÁFICAS}

Arias, E. y Piñeros, P. (2008). Aislamiento e identificación de hongos filamentosos de muestras de suelo de los Paramos de Huasca y Cruz Verde. (Tesis de licenciatura). Pontificia Universidad Javeriana, Bogotá, Colombia.

Buendía, H. (2012) Biorremediación de suelos contaminados por hidrocarburos mediante compost de aserrín y estiércoles. (Tesis de maestría). Universidad Nacional Mayor de San Marcos, Lima, Perú.

Cabanillas, J., y Pissani, V. (2015). Efecto de la bioaumentación y bioestimulación en la eficiencia de biorremediación de suelos contaminados con hidrocarburos de petróleo. (Tesis de licenciatura). Universidad Nacional Pedro Ruiz Gallo, Lambayeque, Perú.

Chaudhry, S., Luhach, J., Sharma, V., y Sharma, C. (2012). Assessment of diesel degrading potential of fungal isoñates from sludge contaminated soil of petroleum refiney, Haryana. Research Journal of Microbiology, 7(3), 182-190.

Corrales, L., Sánchez, L., Cuervo, J., Joya, J., y Márquez, K. (2012). Efecto biocontrolador de Bacillus spp. frente a Fusarium sp. bajo condiciones de invernadero en plantas de tomillo (Thymus vulgaris L.). NovaPublicación Científica en Ciencias Biomédicas, 10(17), 64-82.

Deli, H. (2014). Assessment of the ability of fungi isolates Bipolaris hawaiiensis and Emericella nidulans isolated from soils containing petroleum waste in the analysis of crude oil. Al- Mustansiriyah Journal of Science, 25(3), 7-12.

Flores, S., y Benites, J. (2015). Efecto del estiércol de cuy, porcino y vacuno en la biorremediación de suelo contaminado con hidrocarburos de diésel en terrarios. (Tesis de licenciatura). Universidad Nacional Pedro Ruiz Gallo, Lambayeque, Perú.

Galindo, C., y Llontop, D. (2015). Eficiencia de la biorremediación de suelos contaminados con petróleo por bacterias nativas de la provincia de Talara, región Piura. (Tesis de 
licenciatura). Universidad Nacional Pedro Ruiz Gallo, Lambayeque, Perú.

Hasan, I. (2014). Ability of some soil fungi in biodegradation of petroleum hydrocarbon. Jounal of Applied \& Environmental Microbiology, 2(2), 46-52.

Hasan, I., Mhail, N., y Ali, S. (2016). Evaluation the quality of the oil waste to Al-Nasiriya refinery and possibility of treatment by some filamentous fungi. Advances in Environmental Biology, 10(4), 34-43.

Hernández, E., Ferrera, R., Gutiérrez, M., Rodríguez, R., Rubiños, J., y Fernández, L. $\left(\begin{array}{llll}2 & 0 & 0 & 3\end{array}\right)$. B a c terias y hongos hidrocarbonoclastas de rizósfera frijol y maíz, en un suelo contaminado con petróleo. Terra Latinoamericana, 21(4), 493-502.

Hernández, R., Fernández, C., y Baptista, P. (2014). Metodología de la Investigación ( $6^{\text {ta }}$ ed.). México: Mc Graw, Hill Interamericana Editores S.A.

Llanos, C, (2012). Eficiencia de la biorremediación de suelos contaminados con petróleo por bacterias nativas en el distrito de Pimentel, departamento de Lambayeque, 2011. (Tesis de licenciatura). Universidad Nacional Pedro Ruiz Gallo, Lambayeque, Perú.

Lotfinasabasl, S., Gunale, S., y Rajurkar, N. (2012). Assessment of petroleum hydrocarbon degradation from soil and tarball by fungi. Bioscience Discovery, 3(2), 186-192.

Pernía, B., Demey, R., Inojosa, Y., y NaranjoBriseño, L. (2012). Biodiversidad y potencial hidrocarbonoclástico de hongos aislados de crudo y sus derivados: Un metaanálisis. Revista Latinoamericana de Biotecnología Ambiental y Algal, 3(1), 1-40.

Purisaca, D., y Quevedo, D. (2015). Eficiencia de la biorremediación de suelos contaminados con petróleo por Actinobacterias nativas de la provincia de Talara, región Piura. (Tesis de licenciatura). Universidad Nacional Pedro Ruiz Gallo, Lambayeque, Perú.

Rivera, M., Ferrera, R., Volke, V., Rodriguez, R., y Fernández, L. (2002). Adaptación y selección de microorganismos autóctonos en medios de cultivos enriquecidos con petróleo crudo. Terra, 20, 423-434.

Sandhu, S., Shakya, M., Deshmukh., L, Aharwal, R., y Kumar, S. (2016). Determination of hydrocarbon degrading potentiality of indigenous fungal isolates. International Journal of Environmental Sciences, 6(6), 1163-1172.

Seifert, K. (1996). Fuskey: Fusarium interactive key. Agriculture and agri-food. Canadá.

Tapia, I. (2002). Capacidad degradativa del petróleo de hongos aislados de agua de mar - litoral del Puerto Eten. Enero-Mayo, 2002. (Tesis de licenciatura). Universidad Nacional Pedro Ruiz Gallo, Lambayeque, Perú.

Vanishree, M., Thatheyus, A., y Ramya, D. (2014). Biodegradation of petrol using Aspergillus sp. Annual Research \& Review in Biology, 4(6), 914-923. 